\title{
OPEN-TOP WARMING CHAMBERS REDUCE ANIMAL POLLINATION OF TWO
}

\section{SUBALPINE HERBS}

\author{
Carter P. Adamson ${ }^{1,2, *}$ and Amy M. Iler ${ }^{1,3}$ \\ ${ }^{1}$ Rocky Mountain Biological Laboratory, 8000 County Road 317, Crested Butte, CO 81224 USA \\ ${ }^{2}$ University of California Santa Barbara, Department of Ecology, Evolution, and Marine Biology, Santa Barbara, CA 93106 \\ USA \\ ${ }_{3}^{3}$ Chicago Botanic Garden, the Negaunee Institute for Plant Conservation Science and Action, 1000 Lake Cook Road, \\ Glencoe, IL 60022 USA
}

Journal of Pollination Ecology, 29(17), 2021, pp 231-239

DOI: $10.26786 / 1920-$

$7603(2021) 638$

Received 4 February 2021, accepted 20 August 2021

*Corresponding author: cpadamson@ucsb.edu

\begin{abstract}
Open top chambers (OTCs) are a popular method for studying the biological effects of climate change through passive heating, but their effects on biotic interactions are poorly understood, especially for pollination. Here we use the subalpine plants Delphinium nuttallianum and Potentilla pulcherrima to examine the possibility that the effects of OTCs on plant reproduction are not the result of warming but rather OTCs acting as barriers to pollinator movement. Pollinator observations were conducted and stigmas collected from plants inside and outside of OTCs in a meadow in the Rocky Mountains of Colorado, USA. Very few visitors were observed inside of OTCs, which led to severe reductions in visitation rates, by $92 \%$ in Delphinium and $85 \%$ in Potentilla. The number of conspecific pollen grains on stigmas was $73 \%$ lower in OTCs for Delphinium but not Potentilla, likely because it is capable of autogamous self-pollination. This study clearly shows that OTCs can reduce animal pollination, which is also likely to reduce plant reproductive output of outcrossing plants via decreases in the quantity or quality of pollen. OTCS may therefore confound effects of warming on plant reproduction with pollination effects. Although the unintended effects of OTCs on abiotic conditions are wellstudied, this study highlights that their effects on biotic interactions require further investigation.
\end{abstract}

Keywords-Climate change; experimental warming; OTC; pollen deposition; pollinator visitation

\section{BACKGROUND}

Climate change affects various aspects of plant performance, including survival and reproduction (Hedhly et al. 2009; Miller-Rushing et al. 2010; Cleland et al. 2012). Open top passive warming chambers (hereafter OTCs) are widely used as an experimental manipulation to study how plant performance responds to climate change, because they consistently and passively increase air temperatures in the field (Marion et al. 1997). OTCs have many benefits: they are easy to deploy in remote places and are inexpensive relative to active heating. However, there are also wellknown abiotic side effects, including increased humidity, changes to soil moisture, reduced soil temperature, and reduced wind speeds (Marion et al. 1997; Dabros et al. 2010; Sharkuu et al. 2013). Although the efficacy of OTCs and their abiotic side effects have been studied in a variety of locations, little is known about how OTCs affect plant-animal interactions.

OTCs can increase or decrease rates of insect herbivory depending on the herbivore and the study system (Barrio et al. 2016; Brikemoe et al. 2016), but so far have not been shown to affect plant-pollinator interactions (Robinson \& Henry 2018). However, OTCs have been shown to alter the relative abundances of Lepidoptera, Hymenoptera, and Diptera (Zhang et al. 2015), suggesting that OTCs may affect the composition of pollinator visitors to plants inside chambers. Yet to our knowledge no studies have examined 
whether OTCs affect pollen deposition, along with pollinator behavior. If OTCs act as a physical or visual barrier to pollinators for plants shorter than the OTC, this may decrease the number of visitors and visitation rates to plants inside chambers. Conversely, OTCs might also prevent pollinators from leaving the chambers, which could increase visitation rates if visitors make repeated visits while 'trapped' inside. Similarly, visitors may stay inside chambers if the abiotic conditions inside OTCs are preferable, which could also increase visitation rates. Indeed, OTCs raise air temperatures and reduce wind speeds, conditions that should improve the foraging speed and efficiency of bees (Abrol \& Kapil 1986; Hudson et al. 2011). The only study of which we are aware that directly examines the effect of OTCs on pollinator behavior finds that OTCs have no effect on pollinator visitation rates to three Arctic plant species (Robinson \& Henry 2018). Another study anecdotally reported a qualitative observation that OTCs substantially reduced pollinator visits to an arctic shrub in one year, but not the following year (Marion et al. 1997).

Understanding the effects of OTCs on pollination is especially important for research on how climate change affects the reproduction of plants that require pollinators (e.g., Arft et al. 1999; Cleland et al. 2012). Without explicit consideration of how OTCs affect pollination, the abiotic and biotic drivers of effects of OTCs on plant reproduction may be confounded. This study examines whether OTCs affect the pollination of two subalpine plant species, Delphinium nuttallianum and Potentilla pulcherrima, in the Rocky Mountains, USA. We asked whether OTCs affect: the number of visitors, visitation rates per flower, and the number of conspecific pollen grains deposited on flowers inside OTCs compared to outside of OTCs. We were primarily interested in determining whether OTCs affect visitation and pollen deposition. As a secondary goal, we were interested in examining evidence for whether effects of OTCs on pollination were due to the physical presence of the chamber or due to warming (once visitors were inside the chamber). We expect any effects of OTCs on pollinator behavior to have larger consequences for pollen deposition in Delphinium, which requires pollinators to set seed (Waser \& Price 1981), compared to Potentilla, which is capable of autogamous self-pollination (Burkle \& Irwin 2010).

\section{MATERIALS AND METHODS}

\section{(A) STUDY SITE \& SPECIES}

This study was conducted in a subalpine meadow approximately $4.5 \mathrm{~km} \mathrm{~N}$ of the Rocky Mountain Biological Laboratory (RMBL) in Gothic, Colorado, from June through August of 2019 $\left(107^{\circ} 0^{\prime} 53.806^{\prime \prime} \mathrm{W}, 38^{\circ} 59^{\prime} 39.449^{\prime \prime} \mathrm{N}, 2900 \mathrm{~m}\right.$ above sea level). This area has heavy snow cover for the majority of the year and a three to five month growing season in the summer (CaraDonna et al. 2017).

Both Delphinium nuttallianum (dwarf larkspur) and Potentilla pulcherrima (cinquefoil) are iteroparous perennials. These species were chosen based on their different pollination biology. Delphinium is relatively specialized and Potentilla relatively generalized in their pollination. Delphinium flowers exhibit bilateral symmetry and are mainly pollinated by bumblebee queens and broad-tailed hummingbirds (Waser 1978; Price \& Waser 1979). Potentilla flowers exhibit radial symmetry, are pollinated by a diversity of flies and bees (CaraDonna et al. 2017), and are capable of autogamous self-pollination (Burkle \& Irwin 2010).

\section{(B) EXPERIMENTAL DESIGN}

We conducted pollinator observations and collected stigmas from plants inside and outside of OTCs. The plexiglass OTCs covered an approximately $1 \mathrm{~m}^{2}$ area and measured $58 \mathrm{~cm}$ tall, following the standard design and size for OTCs (Marion et al. 1997; Robinson \& Henry 2018). Each OTC had a $15 \mathrm{~cm}$ wide by $5 \mathrm{~cm}$ tall open window, midway up the south-facing side for increased ventilation (use of the OTCs in previous years showed larger warming effects than desired). The OTCs were attached to the ground with 6" metal landscape staples. OTCs were placed in a meadow where both species were abundant. The flowers of both species were shorter than the height of the OTC panels, and OTCs were set up before plants began to flower. For the first 26 days of the study, data were collected using OTCs from an existing experiment on the effects of climate change on plant phenology and demography. This experiment consisted of $1430 \mathrm{~m}^{2}$ plots containing seven chambers each. Data from $\mathrm{HOBO}$ data 
loggers (Onset Computer Corporation, USA) placed inside these chambers and in adjacent control plots showed that the OTCs warmed mean daytime air temperature (06:00-18:00 hr) by $2.5^{\circ} \mathrm{C}$ during the course of the study. For the remainder of the experiment (13 days), data were collected from a new set of seven total chambers adjacent to the 14 plots, to minimize trampling of plants in the initial plots. These OTCs were set-up after the plants had already begun to flower.

Flowers inside and adjacent to the chambers were observed during 5-min sessions for both sets of plots. Observations outside of chambers occurred within $1 \mathrm{~m}$ of a chamber in an approximately $1 \mathrm{~m}^{2}$ area that was selected to have a similar floral density as the inside of the chamber. All receptive flowers of the focal species present within the observation area were monitored during each observation period. Observations were paired such that a 5-minute observation period for flowers inside a chamber would be followed immediately by a 5-minute observation for nearby flowers outside the chamber in a patch of similar density. The number of flowers being observed, pollinator visitors, and visits were recorded during each session. A visit was defined as a visitor making contact with anthers or stigmas. Over 200 observation sessions were conducted across the whole study. For Delphinium, over 70 observations for each treatment (inside vs. outside of OTCs) were conducted on eight days, over the course of a 27-day period. For Potentilla, over 30 observations for each treatment were conducted on six days, over the course of an 11-day period. Weather data such as cloud cover, wind conditions, and temperature were also recorded, and observations were not conducted on days with precipitation.

Pollen deposition was quantified as the number of conspecific pollen grains per stigma (based on reference slides of each species' pollen). Flowers were first emasculated to ensure that stigma collection did not cause unintended pollination. Stigmas were removed from two flowers of each species inside and outside each of the 7 chambers used in the latter portion of the experiment (14 flowers inside and 14 adjacent to OTCs). All stigmas for each species were removed on the same day. For flowers adjacent to OTCs, it was not noted which chambers were directly adjacent at the time of collection. Instead, it was noted whether stigmas came from one of two larger collection areas surrounding the chambers, so $n=2$ for the control treatment (instead of $n=7$ ). The stigmas were mounted on microscope slides with fuchsin dye and pollen grains were counted under a compound microscope at $40 \mathrm{x}$ magnification. All data and $R$ code will be archived on Dryad upon publication.

\section{(c) ANALYSIS}

All analyses were performed in R Studio using R v. 4.0.2 (R Core Development Team 2020). For each of our response variables (probability of a visitor entering the OTC, visitation rate with zeros, visitation rate without zeros, and number of conspecific pollen grains), model selection was conducted on a candidate set of three models: (1) a species by treatment interaction, (2) species and treatment as additive effects, and (3) an interceptonly null model. The model with the interaction term allowed us to determine whether the effect of OTCs on visitation rates depended on species. The model with the lowest AICc score was selected as the best model using package 'bbmle' (Bolker \& $\mathrm{R}$ Core Development Team 2020). Any significant species by treatment interaction terms were followed up with a one-way ANOVA for each species, to determine whether treatment effects were significant within each species.

The highest number of visitors we observed during an observation session was one, thus our visitor data were 0 's and 1 's, and we treated this response as a binomial response. Thus, we modified our question slightly from 'what is the effect of OTCs on the number of visitors' to 'what is the effect of OTCs on the probability that a visitor enters the chamber?' We used Generalized Linear Mixed Effect Models (GLMMs) with visitor $(0 / 1)$ as the response, a binomial error structure, and date as a random intercept term. The response variable 'probability of a visitor entering the OTC' provides insight into the effects of the physical presence of the chambers, as opposed to effects of warming from the chamber, based on the assumption that a visitor would be unable to detect the warmer temperatures inside the OTC until after entering it.

To analyze pollinator visitation rates (visits per flower per $5 \mathrm{~min}$ ), we used GLMMs with a betabinomial error distribution and date as a random 
intercept term (models with a binomial error distribution were overdispersed). This response variable includes all visitation data, so that visitation rates of zero are also included. Thus, this analysis provides insight into the effect of OTCs on visitation rates, regardless of whether the underlying mechanism is due to the physical presence of the chamber or to warming. This analysis also allows us to connect the results of the probability of entering a chamber with overall visitation rates. It is possible that rates could either differ more than expected between the treatments, or not differ at all, depending on how the visitors behave once entering the chamber.

To determine whether visitation rates differed from those outside of chambers once a pollinator was present, we additionally analyzed visitation rates using only sessions in which a visitor was present (zeros excluded). This analysis provides insight into the effects of whether the conditions inside the OTCs (including warming) affect visitation rates. That being said, there were six observation sessions in which pollinators entered the chambers, so we have a limited ability to detect a difference between treatments due to a low sample size for the chamber treatment. Here we used GLMMs with visitation rate per flower (per 5 $\mathrm{min}$ ) as the response, a binomial error structure, and date as a random intercept term.

For the number of conspecific pollen grains deposited on stigmas, we first calculated the average number of pollen grains per stigma for each plot. We then conducted model selection on linear models, using the candidate set described above.

\section{RESULTS}

Over the course of the study, a total of 40 visitors were observed across 205 observation periods (17 hours and 5 minutes). The average number of Delphinium flowers monitored during each observation period was 21 flowers within chambers and 23 flowers outside of chambers. Similarly, the average number of Potentilla flowers monitored during each observation period was 16 flowers within chambers and 18 flowers outside of chambers. This discrepancy can be attributed to differences in flower density between the two species. We primarily observed bumble bee queens (Bombus spp) on Delphinium and solitary bees and bee flies (Bomblyliidae) on Potentilla. We did not observe hummingbird visits to Delphinium during our observation sessions, but we observed Broad-tailed Hummingbirds foraging on Delphinium in the study area (outside chambers).

The best model for the probability of a visitor entering the OTCs contained treatment and species as additive effects (Table 1, Table 2). OTCs significantly reduced the probability of receiving a visitor in both species, by $81 \%$ for Delphinium (Fig. 1a) and by $88 \%$ for Potentilla (Fig. 1b). We did not find a significant effect of species on the probability of receiving a visit.

Table 1. Model selection tables for the effect of OTCs (open top chambers: treatment) on the probability of a visitor entering the chamber (visitors), the number of visits per flower (visitation rate), and mean number of conspecific pollen grains per stigma. The null model was an intercept-only model. $\mathrm{df}=$ degrees of freedom.

\begin{tabular}{llrr}
\hline response & model & $\Delta$ AICc & $\mathrm{df}$ \\
\hline visitors & species + treatment & 0.0 & 4 \\
& species $\times$ treatment & 1.5 & 5 \\
null & 27.8 & 2 \\
visitation rate & species + treatment & 0.0 & 5 \\
& species $\times$ treatment & 2.0 & 6 \\
visitation rate & null & 29.7 & 3 \\
(visitors present) & null & 0.0 & 2 \\
& species $\times$ treatment & 0.6 & 4 \\
pollen deposition & species + treatment & 2.6 & 5 \\
& species $\times$ treatment & 0.0 & 5 \\
& species + treatment & 8.9 & 4 \\
& null & 14.4 & 2 \\
\hline
\end{tabular}


Table 2. Effects of OTCs on the probability of a visitor entering the chamber (visitors), pollinator visitation rate (\# visits per flower per $5 \mathrm{~min}$ ), and pollen deposition (mean \# conspecific grains per stigma) to two subalpine plant species, Delphinium nuttallianum and Potentilla pulcherrima. Treatment: OTCs present or absent. The test statistic is a z-value for visitors and visitation rate, and a t-value for pollen grains.

\begin{tabular}{llrrr}
\hline Response & Predictor & Estimate \pm 1 SEM & test statistic & p-value \\
\hline visitors & Intercept & $-3.07 \pm 0.46$ & -6.66 & $<0.0001$ \\
& Treatment & $2.25 \pm 0.48$ & 4.73 & $<0.0001$ \\
& Species & $0.62 \pm 0.41$ & 1.53 & 0.13 \\
\multirow{4}{*}{ visitation rate } & Intercept & $-5.34 \pm 0.54$ & -9.90 & $<0.0001$ \\
& Treatment & $2.35 \pm 0.53$ & 4.40 & $<0.0001$ \\
& Species & $0.50 \pm 0.35$ & 1.45 & 0.15 \\
& & $6.46 \pm 1.49$ & 4.33 & 0.0007 \\
& Intercept & $18.93 \pm 3.17$ & 5.98 & $<0.0001$ \\
& Treatment & $7.90 \pm 2.11$ & 3.74 & 0.0022 \\
& Species & $-17.03 \pm 4.48$ & -3.80 & 0.0019 \\
\hline
\end{tabular}

Delphinium nuttallianum
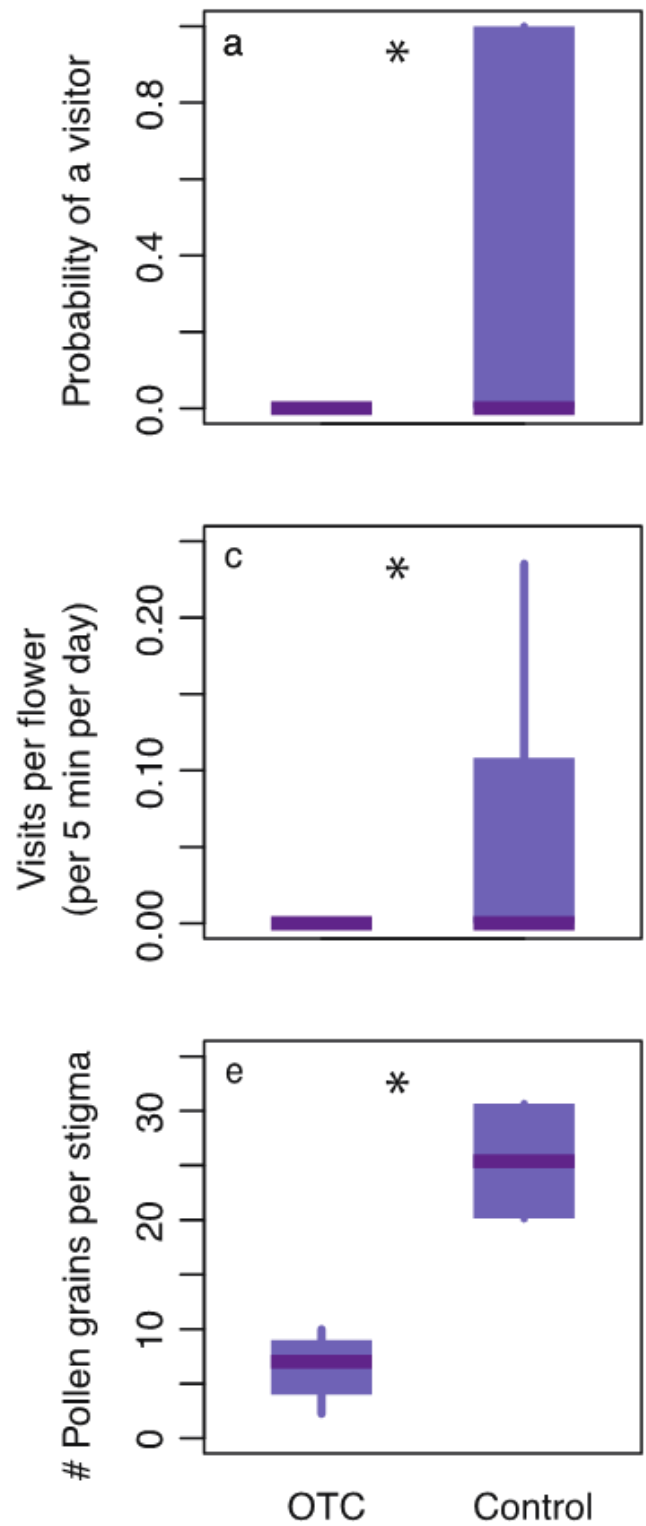

\section{Potentilla pulcherrima}
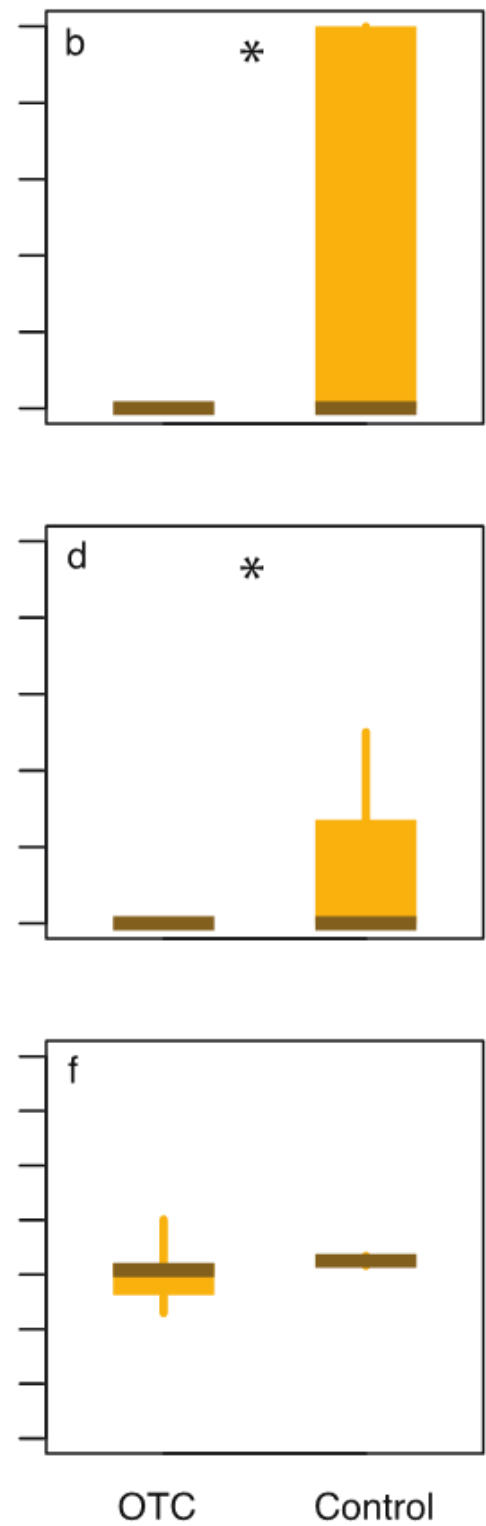

Figure 1. Effects of passive open top warming chambers (OTCs) on pollinator visitation (a-d) and pollen deposition $(e, f)$ to two subalpine herbs: Delphinium nuttallianum and Potentilla pulcherrima. Box plots represent the range of data for each species and treatment. For each box, the central line is the median, the edges of the box are the $25^{\text {th }}$ and $75^{\text {th }}$ quartiles, and the lines represent the $5^{\text {th }}$ and $95^{\text {th }}$ quartiles. Note that panels ab were analyzed with a betabinomial and c-d with a binomial error distribution. Asterisks denote significant differences at $P<0.05$. 
The best model for visitation rate contained treatment and species as additive effects (Table 1). The OTCs significantly reduced visitation rates for both species, by $92 \%$ in Delphinium and $85 \%$ in Potentilla (Table 2; Fig. 1c, d). We did not find a significant effect of species on visitation rates. In contrast, when visitation rates are limited to observation sessions in which we observed a visitor, the null model was best (the next best model was 0.6 AIC units away; Table 1). Thus, we did not detect a significant effect of OTCs or species on visitation rates once a visitor was present (Fig. 2).

The best model for pollen deposition included a species by treatment interaction $\left(R^{2}=0.75, F_{3,14}=\right.$ 13.66, $P=0.0002$; Table 1, Table 2). OTCs significantly reduced the mean number of conspecific pollen grains on Delphinium stigmas by $73 \%$ (one-way ANOVA: $F_{1,7}=34.73, P=0.0006$ ); in contrast, we did not detect a significant effect of OTCs on pollen deposition to Potentilla (one-way ANOVA $F_{1,7}=0.37, P=0.56$; Fig. 1 e, f).

\section{Discussion}

Here we show that OTCs can substantially reduce both pollinator visitation and pollen deposition in a subalpine ecosystem. We find reductions in the number of visitors and therefore visitation rates to both species, in addition to reduced pollen deposition in Delphinium. OTCs did not appear to reduce pollen deposition in Potentilla, likely due to its capacity for autogamous self-pollination (Burkle \& Irwin 2010). The effects of artificial warming chambers on plant-pollinator interactions are poorly understood, despite the fact that such chambers are frequently used to study the effects of warming on plant reproduction (Arft et al. 1999; Cleland et al. 2012). Given that our study shows that OTCs can affect pollinator behavior and pollen deposition, future studies that employ OTCs to study effects of warming on plant reprouction should take potential effects of the chambers on pollination into consideration.

Lower visitation rates inside OTCs appear to be caused by a reduced likelihood for pollinators to enter the chambers, rather than fewer visits once a pollinator enters the chambers. The median number of visitors inside OTCs was zero for both plant species, and in cases when a visitor did arrive, the average visitation rate to flowers does not differ between treatments. This may be due to potential pollinators detecting the chambers and simply avoiding the unknown objects while foraging. Alternatively, it may be more energetically efficient for pollinators to maintain their foraging height while visiting flowers outside of the chambers than to alter their foraging height to access flowers within chambers. Many pollinators exhibit a preferred height range within which they tend to forage, a phenomenon referred to as foraging height constancy (Levin \& Kerster 1973; Faulkner 1976; Levin \& Watkins 1984). A previous study at the RMBL observed foraging height constancy in bumblebees, solitary bees, and hummingbirds on Potentilla and a congener of $D$. nuttallianum, D. barbeyi (Waddington 1979).
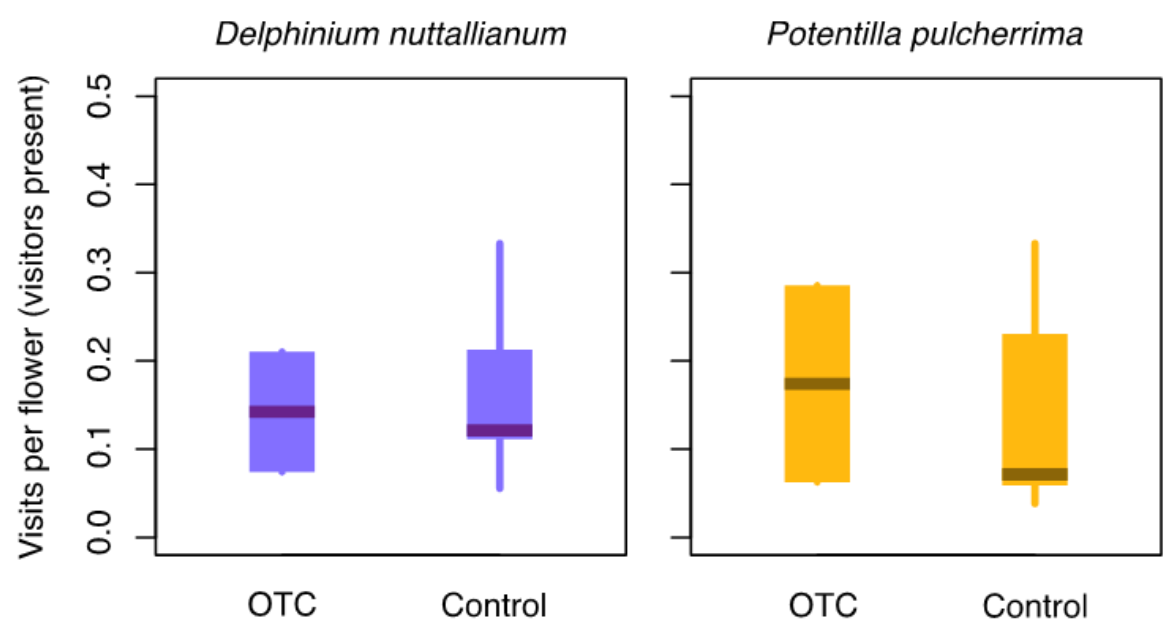

Figure 2. Visitation rates to both species for observation sessions in which at least one pollinator was present. Species are not shown separately because the null model was the best model (no treatment or species effects). Box plots represent the range of data for each treatment. The central line is the median, the edges of the box are the $25^{\text {th }}$ and $75^{\text {th }}$ quartiles, and the lines represent the $5^{\text {th }}$ and $95^{\text {th }}$ quartiles. 
Pollination could be impacted by OTCs if flowering stalks are shorter than the chamber panels, as they were in this experiment, thus forcing prospective pollinators to increase their foraging height to rise over and into the chamber in order to access the flowers inside. Future work could further address the hypothesis that OTCs act as a physical barrier by making chambers shorter to allow focal flowers to protrude above the chamber, or by making windows in the chamber through which pollinators can enter. A single window large enough for insects to pass through (15 $\mathrm{cm}$ wide by $5 \mathrm{~cm}$ tall) was present on the chambers used in this experiment (to reduce the magnitude of warming), but we still find large reductions in visitors and visitation rates. Thus, to be effective, windows would likely need to be larger and strategically located at the foraging heights of pollinators. Shorter chambers and windows are both likely to reduce warming effects (Marion et al. 1997), creating a trade-off between less warming vs. reducing the effects of OTCs on pollinators.

The altered abiotic environment within chambers is likely to affect pollinators once they enter the chamber. Although our sample size was small, we do not find support for this hypothesis. We do not detect an effect of OTCs on visitation rates for observation sessions in which a pollinator was present, indicating that altered abiotic factors within the chamber such as increased temperature and reduced wind speed did not have detectable effects on pollinator activity; the altered pollinator behavior observed in this study is therefore more likely explained by the physical barrier of the chambers themselves, rather than the conditions within. However, more data on visitation rates inside chambers might reveal an increase in visitation rates compared to controls, based on the direction of the chamber effect (Fig. 2), because few visitors entered the OTCs in our study. To directly separate effects of the chambers' physical presence from effects on the abiotic environment, future studies could create a treatment with dummy chambers that do not affect environmental conditions: mesh structures that minimally affect air temperature and wind speeds while still providing a physical barrier, or OTCs with numerous holes in the plexiglass covered by mesh, so that wind can flow through the chambers while preventing pollinator access. Another future direction of interest would be to emasculate all study flowers, to determine the effects of OTCs on outcross pollen deposition.

One caveat to our study is that OTCs advance the flowering date of both species in our study (Jerome, 2020; Jerome \& Iler, unpublished data). Thus, for the first half of the experiment that used OTCs that were set up before plants began to flower, visitors may have been responding to altered flowering phenology rather than the presence of the OTC. However, this seems unlikely because there would have been slightly more flowers in the OTCs compared to the controls during this time of the study. Thus, we would expect pollinators to be attracted to the higher flowering densities inside the chambers and show a bias towards those flowers. This is the opposite of what we found, suggesting that the pollinators react more strongly to the presence of the OTC than to the advanced flowering date. The OTCs should not have affected flowering phenology in the second half of the experiment, because they were set up after plants began to flower.

We find consistent reductions in visitors and visitation rates in OTCs despite differences in the identity of the primary pollinators of our two study species, suggesting that OTCs may affect a wide range of pollinator taxa. However, some pollinator taxa may not be affected by the presence of OTCs; a study in the Arctic finds no evidence that OTCs affect pollinator visitation rates (Robinson \& Henry 2018). The primary pollinators in our study were bumble bees, solitary bees, and bombyliid flies, whereas $99 \%$ of visits in the Arctic study were from different fly taxa (primarily anthomyiid, muscid, and syrphid flies). The size and design of OTCs used in Robinson \& Henry (2018) is analagous to that used here (following the International Tundra Experiment), so the differences between studies are unlikely due to different chamber designs. It has been noted that the Arctic has a high proportion of generalist pollinators (Elberling \& Olesen 1999). It may be possible that generalists that visit multiple flower species with different heights exhibit a lesser degree of foraging height constancy when compared to other pollinator communities that include generalists and specialists. In such a case, the higher proportion of generalists in the Arctic 
might be less affected by the physical barrier of OTC panels and more disposed to simply fly over them, but this hypothesis requires further research.

Our results suggest that OTCs might disproportionately affect the reproductive success of species that require animal pollination to successfully reproduce (Delphinium) over species capable of selfing (Potentilla). Delphinium nuttallianum has been shown to be pollen limited in this general study area (Burkle et al. 2007), suggesting that reductions in pollen deposition in the OTCs could reduce seed production. Although fewer visitors and lower visitation rates could still reduce seed production in Potentilla, because female reproductive success is higher when these plants receive outcross vs. self pollen (Vail 1983), this seems unlikely given that its reproduction does not appear to be pollen limited in this general study area (Burkle \& Irwin 2010). Consistent with this interpretation, another study that used the same OTCs as used in the first half of this study found reduced seed production in Delphinium plants inside the chambers compared to plants outside, but no significant change in seed production was observed between these two treatments in Potentilla (Jerome 2020; Jerome et al. in review). However, the effects of reduced pollination cannot be separated from the direct effects of warming on seed production in this case. Our results highlight this potential risk of confounding the direct effects of passive warming by OTCs on plant reproduction with the unintended effects of OTCs on pollination.

The interpretation of experiments using OTCs to study plant responses to climate change may consequently be invalid if the unintended effects of OTCs on pollinators are not carefully considered. In theory, the unintended consequences of OTCs could extend beyond plant reproduction, especially for multi-year studies of perennial plants. If plants experience reduced pollination and reproduction, then they are likely to allocate more resources to vegetative growth and survival (i.e., costs of reproduction; reviewed in Obeso 2002). Thus, increased plant growth inside of chambers could in theory reflect indirect effects of low pollination and reallocation of resources instead of a direct response to warming, but this requires much further study. Like the effects of OTCs on the abiotic environment, the effects of OTCs on pollinators will likely vary greatly by study site and species. Because OTCs are a popular method for examining plant responses to climate change, additional research on how OTCs affect interactions between plants within the chamber and other organisms will improve our understanding of biological responses to climate change.

\section{ACKNOWLEDGEMENTS}

Thanks to N. Waser and M. Price for insightful feedback on the study and the use of laboratory equipment. The authors also thank the Iler-CaraDonna lab for useful feedback on an earlier draft of the paper.

This study was funded by an undergraduate research fellowship from the Rocky Mountain Biological Laboratory (awarded to CPA).

\section{AUTHORS' CONTRIBUTIONS}

AMI conceived the study, CPA conducted fieldwork, AMI and CPA analyzed data, CPA wrote the paper, and AMI edited multiple drafts.

\section{DATA ACCESSIBILITY}

Data sets and code: Dryad doi:10.25349/D9KK6Z

\section{REFERENCES}

Abrol DP, Kapil RP (1986) Factors affecting pollination activity of Megachile lanata Lepel. Proceedings: Animal Sciences 95:757-769.

Arft AM, Walker MD, Gurevitch JEA, Alatalo JM, BretHarte MS, et al. (1999) Responses of tundra plants to experimental warming: meta-analysis of the international tundra experiment. Ecologial Monographs 69:491-511.

Barrio IC, Bueno CG, Hik DS (2016) Warming the tundra: reciprocal responses of invertebrate herbivores and plants. Oikos 125:20-28.

Birkemoe T, Bergmann S, Hasle TE, Klanderud K (2016) Experimental warming increases herbivory by leafchewing insects in an alpine plant community. Ecology and Evolution 6:6955-6962.

Bolker B, R Core Development Team (2020) bbmle: Tools for general maximum likelihood estimation. [online] URL: http://CRAN.Rproject.org/package=bbmle

Burkle LA, Irwin RE (2010) Beyond biomass: measuring the effects of community-level nitrogen enrichment on floral traits, pollinator visitation and plant reproduction. Journal of Ecology 98:705-717.

CaraDonna PJ, Petry WK, Brennan RM, Cunningham JL, Bronstein JL, Waser NM, Sanders NJ (2017) Interaction 
rewiring and the rapid turnover of plant-pollinator networks. Ecology Letters 20:385-394.

Cleland EE, Allen JM, Crimmins TM, Dunne JA, Pau S, Travers SE, Zavaleta ES, Wolkovich EM (2012) Phenological tracking enables positive species responses to climate change. Ecology 93:1765-1771.

Dabros A, Fyles JW, Strachan IB (2010) Effects of opentop chambers on physical properties of air and soil at post-disturbance sites in northwestern Quebec. Plant and Soil 333:203-218.

Elberling H, Olesen JM (1999) The structure of a high latitude plant-flower visitor system: the dominance of flies. Ecography 22:314-323.

Faulkner GJ (1976) Honeybee Behaviour as Affected by Plant Height and Flower Colour in Brussels Sprouts. Journal of Apicultural Research 15:15-18.

Hedhly A, Hormaza JI, Herrero M (2009) Global warming and sexual plant reproduction. Trends in Plant Science 14:30-36.

Hudson JMG, Henry GHR, Cornwell WK (2011) Taller and larger: shifts in Arctic tundra leaf traits after 16 years of experimental warming. Global Change Biology 17:1013-1021.

Jerome DK (2020) Early snowmelt and warming independently drive the reproductive phenology of subalpine wildflowers. Masters Thesis, Northwestern University.

Levin DA, Kerster HW (1973) Assortative Pollination for Stature in Lythrum salicaria. Evolution 27:144-152.

Levin DA, Watkins L (1984) Assortative mating in phlox. Heredity 53:595-602.

Marion GM, Henry GHR, Freckman DW, Johnstone J, Jones G, Jones MH, Lévesque E, Molau U, Mølgaard P, Parsons AN, Svoboda J, Virginia RA (1997) Open-top designs for manipulating field temperature in highlatitude ecosystems. Global Change Biology 3:20-32.

Miller-Rushing AJ, Høye TT, Inouye DW, Post E (2010) The effects of phenological mismatches on demography. Philosophical Transactions of the Royal Society B: Biological Sciences 365:3177-3186.

Obeso JR (2002) The costs of reproduction in plants. New Phytologist 155:321-348.

Price MV, Waser NM (1979) Pollen dispersal and optimal outcrossing in Delphinium nelsoni. Nature 277:294-297.

R Core Development Team (2016) R: A language and environment for statistical computing. R Foundation for Statistical Computing. Vienna, Austria. [online] URL: https://www.R-project.org

Robinson SVJ, Henry GHR (2018) High Arctic plants show independent responses to pollination and experimental warming. Botany 96:385-396.

Sharkhuu A, Plante AF, Enkhmandal O, Casper BB, Helliker BR, Boldgiv B, Petraitis PS (2013) Effects of open-top passive warming chambers on soil respiration in the semi-arid steppe to taiga forest transition zone in Northern Mongolia. Biogeochemistry 115:333-348.

Vail SG (1983) Density effects in the plant-animal interactions of Potentilla gracilis Douglas (Rosaceae). Masters Thesis, University of Maryland

Waddington KD (1979) Divergence in inflorescence height: an evolutionary response to pollinator fidelity. Oecologia 40:43-50.

Waser NM (1978) Competition for hummingbird pollination and sequential flowering in two Colorado wildflowers. Ecology 59:934-944.

Waser NM, Price MV (1981) Pollinator Choice and Stabilizing Selection for Flower Color in Delphinium nelsonii. Evolution 35:376-390.

Zhang C, Wang L-L, Yang Y-P, Duan Y-W (2015) Flower evolution of alpine forbs in the open top chambers (OTCs) from the Qinghai-Tibet Plateau. Scientific Reports 5:10254. 